\title{
The Ubiquitous TB Otitis Media: A Review of Diagnosis and Treatment Outcomes
}

\author{
Generoso T. Abes, ${ }^{1,2}$ Franco Louie LB. Abes ${ }^{2}$ and Teresa Luisa I. Gloria-Cruz ${ }^{1,2}$ \\ ${ }^{1}$ Philippine National Ear Institute, National Institutes of Health, University of the Philippines Manila \\ ${ }^{2}$ Department of Otorhinolaryngology, College of Medicine and Philippine General Hospital, University of the Philippines Manila
}

\begin{abstract}
Objective. The purpose of this review is to improve the detection of TB otitis media cases and its treatment outcome by describing the varied clinical presentations, discussing the importance, limitations and frontiers of possible diagnostic tests and illustrating the roles of medical and surgical interventions.
\end{abstract}

Method. A review of available literature was done. The search included published researches on TB Otitis Media and related articles on tuberculosis.

Results. The review included aspects of the clinical presentation, diagnostic considerations, and treatment options, both medical and surgical, of tuberculous otitis media.

Conclusion. Tuberculous otitis media presents with a variety of clinical features so that a high index of suspicion is very important. Diagnostic examinations are available but are varied depending on the clinical presentation. Definitive treatment is mainly medical with anti- Koch's medications.

Key Words: tuberculous ear, tb ear, tb otitis media, diagnosis of tb otitis media

\section{Introduction}

Tuberculosis (TB) was considered a major health problem since the beginning of civilization and will probably remain so until disease bothers men. TB was referred to as "phthisis" by Hippocrates (460-377BC), which means "to consume" or "to waste away". Compared to other acute epidemics that affected men like smallpox, typhus, plague and yellow fever, TB has been an ever present health problem. The disease was named "tuberculosis" by Johan Schoenlein in 1839, to refer to the Latin word "tubercula" (the diminutive of "tuber"), which means a small lump. It was thought to be present in animals, especially cattle, and from whom men probably acquired the disease. In 1913, Moore of Cornell University wrote "As a destroyer of man, tuberculosis has no equal; as a scourge of cattle, there is no other to compare $i^{\prime \prime} .^{1}$

Corresponding author: Teresa Luisa I. Gloria-Cruz, MD

Department of Otorhinolaryngology

Philippine General Hospital

University of the Philippines Manila

Taft Avenue, Ermita, Manila 1000 Philippines

Telephone: +6325264360

Email: techgloriacruz@yahoo.com
It continues to be a significant cause of morbidity and mortality despite new developments in diagnosis and aggressive TB prevention and treatment programs. ${ }^{2}$ New cases of TB were reportedly brought about by the increasing incidence of HIV infection particularly in some developing countries, $^{3}$ transmission of the disease from foreign-born immigrants who were considered potential carriers of the disease, ${ }^{4}$ rise of drug-resistant TB, decline of TB control programs in some areas, and increased transmission of infection, particularly in institutional settings. ${ }^{5}$

Extrapulmonary tuberculosis accounts for $15-20 \%$ of tuberculosis in populations with a low prevalence of HIV infection. ${ }^{6}$ Tuberculous otitis media (TBOM) is not listed as a common form of extrapulmonary TB. ${ }^{7}$ It is neither a frequent cause of head and neck mycobacterial infection. 8,9 Presently TBOM is a rare diagnosis ${ }^{10}$ despite its increased incidence of all forms of TB even in the developed world. ${ }^{11}$ Though TBOM was recognized as a distinct disease entity by the detection of the TB bacilli in the ear discharge just three years after clinical TB was described in $1853,{ }^{12}$ and several years before Koch's discovery of the tubercle bacilli in $1882,{ }^{13}$ early detection of the disease remains a difficult challenge for the clinician. ${ }^{14}$ One of the early accounts of TBOM was provided by Turner and Fraser in 1915 who reported that TB was the cause of suppurative otitis media in $50 \%$ of infants under the age of one year and in $27.9 \%$ under the age of 2 years. ${ }^{15}$ It was a common cause of middle ear infection during the first half of the twentieth century, being less common in older children and adults. ${ }^{16}$ Due to the introduction of effective anti-tuberculous drugs, effective milk pasteurization methods, BCG vaccination and improved health service delivery programs, ${ }^{17,18}$ the incidence of tuberculous otitis media dropped to less than one percent in the second half of the second century. ${ }^{19,20}$ The bacterium's ability to survive in a latent state, thereby serving as a reservoir for potential reactivation, ${ }^{3}$ is considered to be an explanation for its characteristic ubiquity. However, it fails to explain the corresponding low incidence of TBOM, ${ }^{10}$ as evidenced by the few sporadic reports in the literature. The rarity of the diagnosis itself, the lack of awareness of its varied clinical presentations, and the ability of TBOM to mimic the signs and symptoms of the more common non-tuberculous otitis media ${ }^{14}$ and other 
mycobacterial infections explain its tendency to be overlooked as a possible diagnosis.

The purpose of this review is to improve the detection of TB otitis media cases and its treatment outcome by describing the varied clinical presentations, discussing the importance, limitations and frontiers of possible diagnostic tests and illustrating the roles of medical and surgical interventions.

\section{Method}

A review of available literature was done. The search included published researches on TB Otitis Media and related articles on tuberculosis.

\section{Results}

\section{Review of the clinical presentations}

Traditional teachings suggest that the typical clinical presentation of TBOM consist of multiple eardrum perforations, which is due to the presence of multiple granulomas in the lamina propria of the tympanic membrane. ${ }^{21}$ Soon after, these multiple perforations will coalesce and form a single large eardrum perforation. ${ }^{16}$ Hence, the occurrence of multiple perforations is an early transient event in the pathophysiology of middle ear TB and may partly explain the reason why it is encountered in only approximately $5 \%$ of cases today. ${ }^{22}$ Later reports mention the combination of painless, persistent otorrhea that is resistant to antibiotic treatment, ${ }^{16}$ early severe hearing loss, facial nerve paralysis in young patients and children, ${ }^{23}$ and complications related to bone necrosis such as postauricular fistula, labyrinthitis and osteomyelitis of the petrous pyramid, as indicative of TBOM. ${ }^{16}$ It is believed that TBOM may be differentiated from the more common nontuberculous middle ear infections by the absence of pain and fever, and by its insidious onset. ${ }^{24}$ Otalgia is considered an infrequent complaint, probably attributed to rupture of the multiple tubercles in the tympanic membrane and seen in less than twenty percent of cases. ${ }^{16,23}$ It is therefore not considered a reliable sign of TBOM. Otorrhea is variably observed in some case series ${ }^{22,23}$ but it is generally noted to be refractory to standard treatment for chronic otitis media. ${ }^{22}$ Four of twelve ears reported in a local study had otorrhea as the primary presenting complaint with symptom duration ranging from twelve to thirty six months prior to diagnosis (unreported data). Dizziness was a more common complaint of patients in earlier reports and its occurrence was explained on the basis of labyrinthitis that may be masked by the gradual course of the disease. ${ }^{24}$ Facial nerve paralysis, a common complication prior to the advent of antituberculous therapy, is described in $15 \%$ to $40 \%$ of TBOM cases $^{22}$ and mostly in children. ${ }^{16,23}$ It was earlier thought that the incidence of facial nerve paralysis was relatively higher than would be expected in non-tuberculous otitis media and its presence, particularly in children, was considered highly indicative of a tuberculous infection. ${ }^{16,23}$ Of the twelve patients in our local series, none presented with facial paralysis but two patients complained of taste abnormality which is considered as an early sign of facial nerve dysfunction. ${ }^{20}$ Meningitis is considered a less common complication, found in 2 of 23 patients in one study, and is thought to be due to hematogenous rather than direct intracranial extension. ${ }^{16}$ However, a recent study found a higher incidence of TB otomastoiditis (34.3\%) among patients who were diagnosed with TB meningitis, indicating that although the exact causal relationship remains unclear, TB meningitis is frequently accompanied by TB otomastoiditis. ${ }^{25}$

Contemporary studies indicate that TBOM has no typical clinical presentation. In fact, it can have variable and insidious features with a chronic, indolent course. ${ }^{10}$ Like the earlier reported cases, contemporary cases are likely to be mistaken for the more common purulent otitis media. ${ }^{13,14}$ Earlier reports included mostly children living in low socioeconomic environments and highly endemic areas, ${ }^{16}$ but contemporary cases were also found among apparently healthy adult patients, ${ }^{22,23}$ some were immigrants ${ }^{10}$ and professionals with suspected exposure to TB cases. ${ }^{14,26}$ Though cases with tympanic membrane perforation with ear discharge and exuberant granulation tissues are still found, more diverse clinical presentations were identified in a local study. ${ }^{14}$ Of the 12 cases (13 ears) seen within a period of five years (2004-2009), only three of five ears were discharging ears with granulation tissue formation; whereas, the other two presented with minimal discharge without granulation tissue formation. The remaining eight ears presented with intact tympanic membranes - four with otitis media with effusion and the other four with thickened tympanic membrane and mass-like lesion in the middle ear (Figure 1). These clinical manifestations are therefore not in accordance with classical descriptions, similar to the observation of several investigators. ${ }^{22}$ From the foregoing data, it appears that the clinical spectrum of TBOM is quite similar to that of the more common, non-tuberculous purulent otitis media (POM) except that in acute purulent otitis media, the initial symptoms frequently consist of fever, otalgia and other acute signs. On the other hand, the clinical course of TBOM is more insidious. Both may, however, be manifested in their early course by signs of middle ear inflammation including the presence of middle ear effusion for which antibiotic may be prescribed. Though some cases of POM may be similarly unresponsive to antibiotic and may cause dull eardrum, TBOM cases were noted to cause thickened TM with masslike lesion in the middle ear. Such clinical course is not commonly observed in non-TB otitis media and should prompt the clinician to pursue investigations to rule out TBOM. 


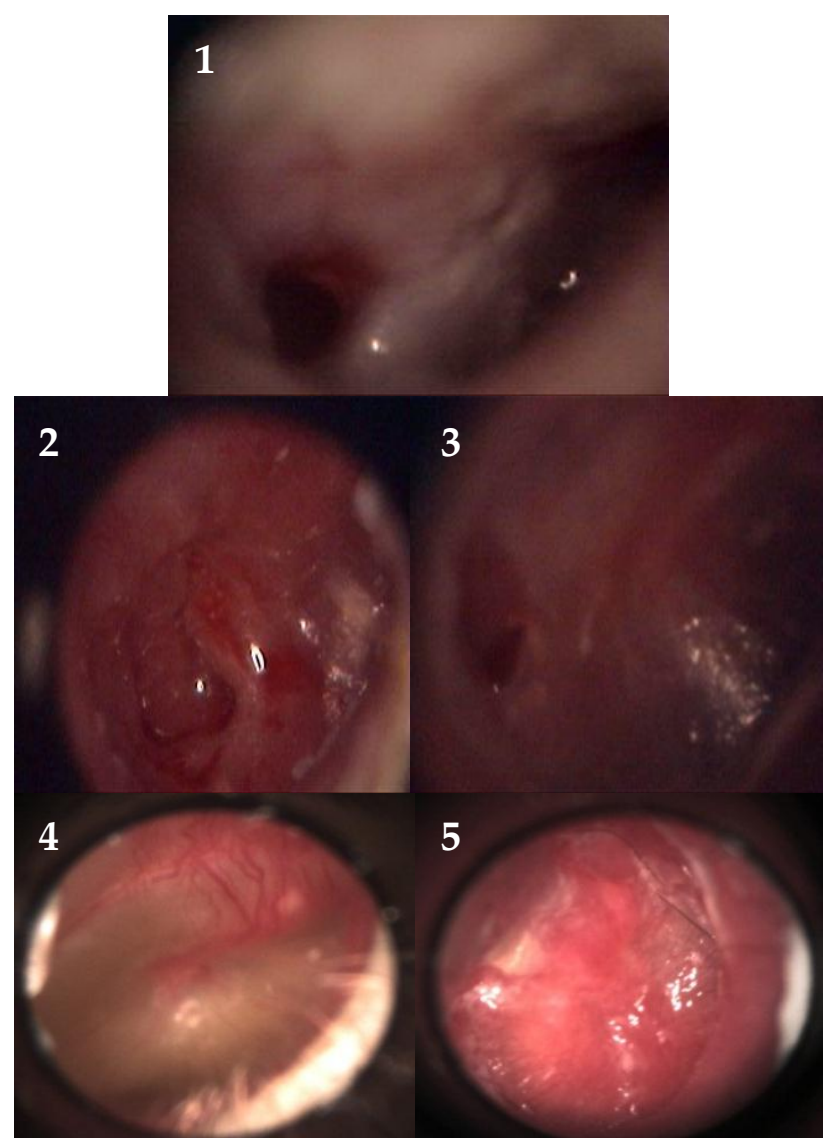

Figure 1. Five clinical patterns identified in our local study.

1. Multiple eardrum perforations

2. Single eardrum perforation, granulation tissue formation, otorrhea refractory to treatment

3. Single eardrum perforation, no granulation tissue formation, refractory otorrhea

4. Intact tympanic membrane, middle ear effusion

5. Intact eardrum, mass-like lesion in middle ear cavity

Earlier studies observed hearing loss to be mostly of the mixed type, severe degree and was thought to be dependent on the degree of inner ear involvement ${ }^{22}$ and inflammatory cell infiltration of the cochlear nerve. ${ }^{27}$ Contemporary cases, however, tend to be of the conductive type, although mixed type may also be encountered, and, may be mild to profound in severity. ${ }^{10,14}$ The degree and type of hearing loss may be associated with the severity of middle and inner ear involvement at the time of diagnosis, hence, highly variable (Figure 2). ${ }^{10}$ Hearing loss was either the primary or an associated symptom in almost all twelve patients in our local series with an average symptom duration of 3 months prior to diagnosis. ${ }^{28}$ In fact, patients who had unexplained hearing loss after adequate treatment were more likely to be investigated earlier for TBOM than those whose main complaint was otorrhea.

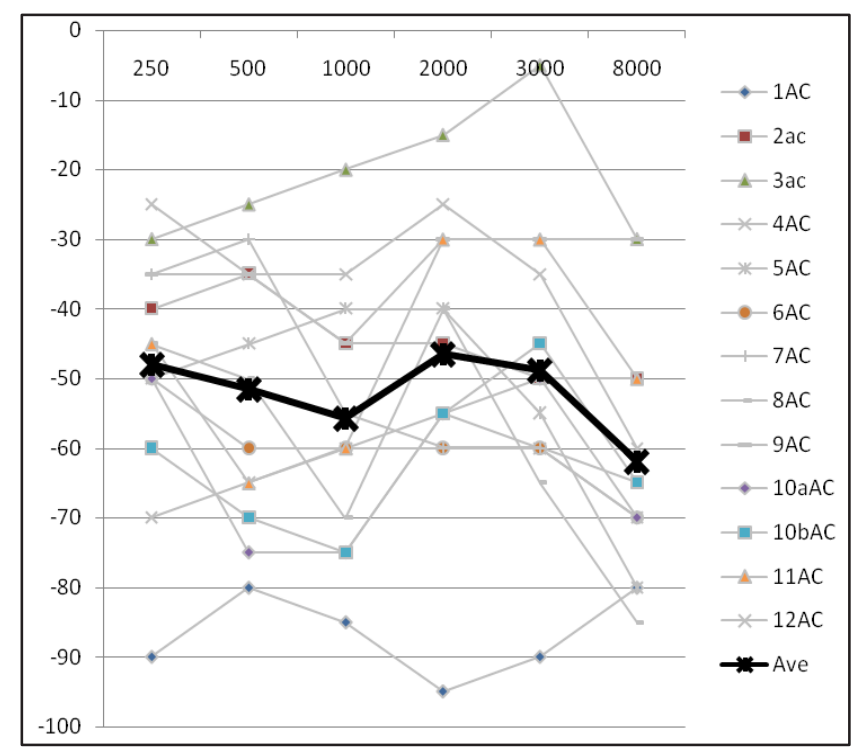

A

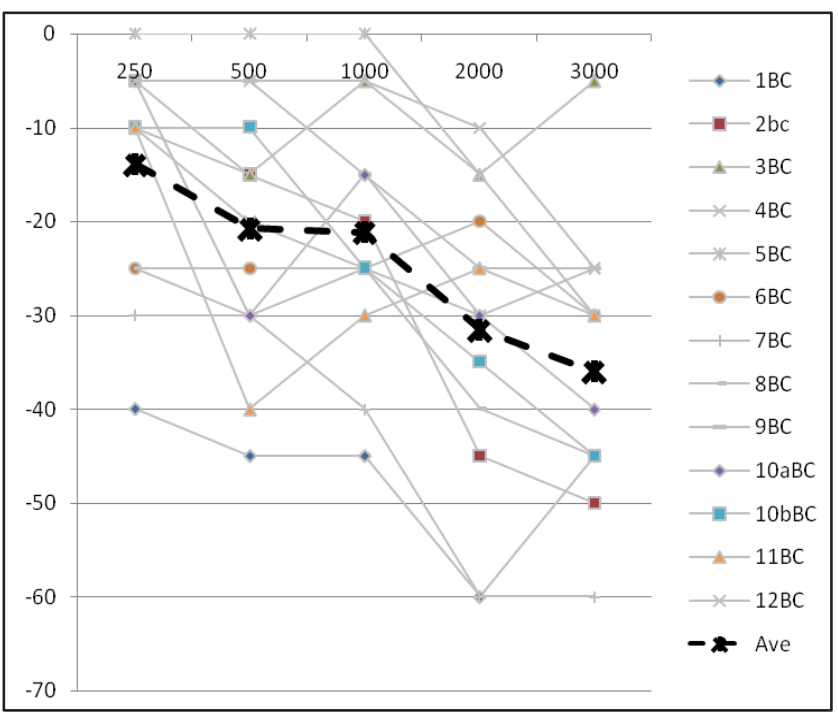

B

Figure 2. Wide variability of pre-treatment air and bone conduction thresholds in thirteen ears. The individual air conduction thresholds are represented by the light lines and the average values by the heavy lines (A). Correspondingly, the bone conduction thresholds are represented in B.

The foregoing observations indicate that besides its rarity, the wide diversity in its clinical presentation notably explain the difficulty in diagnosing TBOM. The variable signs and symptoms confirm the belief that TBOM cannot be diagnosed solely on the basis of clinical manifestations ${ }^{22}$ and, that a high index of suspicion is necessary in order to confirm the presence of the disease. ${ }^{14}$ The clinical challenge is to identify the clinical characteristics that should prompt the clinician to pursue the necessary confirmatory tests. 


\section{Review of the diagnosis of TB Otitis Media}

The diagnosis of tuberculosis, whether pulmonary or extrapulmonary, depends as much on careful history and clinical examination as on diagnostic tests to confirm the presence of the disease. ${ }^{6}$ Items in the clinical history and physical examination may raise the index of suspicion for TB otitis media and may prompt the clinician in pursuing the relevant diagnostic test(s). In our local study ${ }^{14}$ these items in the clinical history were non-responsiveness to standard therapy for purulent, non-bacterial otitis media, history of possible exposure to TB infected individual, past history of pulmonary $\mathrm{TB}$, and recent onset of hearing loss. Lack of responsiveness to standard therapy for otitis media including the use of antibiotics, whether topical or systemic, has been consistently reported to be an indication for suspecting tuberculous otitis media. ${ }^{13,25,29}$ One of the cases included in the local series ${ }^{14}$ was even given intravenous amoxicillin plus clavulanic acid prior to the decision to submit the patient to surgery, which nevertheless resulted to failure in the relief of ear discharge. Another patient was given several weeks of oral amoxicillin plus clavulanic acid for four weeks and had myringotomy with ventilation tube insertion which resulted to the extrusion of the ventilation tube after less than a month. A repeat procedure was done which gave the same result, during which time a mass-like lesion was noted behind the intact tympanic membrane (Figure 2). The otoscopic appearance of TBOM is quite indistinguishable from purulent, non-tuberculous otitis media except for two aspects - non-responsiveness to standard treatment and the presence of mass-like lesion in the middle ear. Non-responsiveness to standard treatment in purulent otitis media is usually due to the presence of cholesteatoma, cholesterol granuloma and irreversible mucosal pathology. Presence of mass-like lesion in the middle ear, particularly when identified on serial otoscopic examination, may connote the presence of middle ear granuloma particularly when the initial clinical features suggest an infectious process. Thus, absence of these two clinical features - non-responsiveness to standard therapy for otitis media and mass-like middle ear lesion, especially if noted after a period of middle ear effusion - may therefore be considered indicative of TBOM.

A history of possible exposure or $\mathrm{TB}$ contamination from infected individuals should arouse suspicion about the possibility of TBOM. TB otitis media is even considered a professional disease in one article ${ }^{26}$ because of the perceived increased probability of acquiring the disease in adults who are at risk of contamination from patients or during handling of specimen for TB. Seven of twelve patients in the local series - an information technologist in a TB institute, a social worker, a community worker, a hospital employee, a medical technologist, a nurse and a scuba diver - had possible $\mathrm{TB}$ infection in relation to the practice of their respective professions. ${ }^{14}$ Transmission of TB begins with the production of the infectious particle by any activity involving forced expiration including coughing, sneezing, singing or breathing. ${ }^{3}$ It is quite understandable how TB transmission may be possible among professionals exposed to TB cases and how a scuba diver instructor could possibly acquire TB infection in the course of conducting courses by sharing a common diving mask with an infected student. Since the route of infection may start in the nasopharynx, a concurrent granulomatous lesion may be seen as experienced in one patient in our study. ${ }^{14}$ Hence, a nasopharyngeal examination, whether by indirect or endoscopic examination, should be routinely done particularly in a patient presenting with a unilateral otitis media with effusion.

High resolution CT (HRCT) of the temporal bone is commonly used in the evaluation of chronic suppurative otitis media because of its value in detecting bone erosion and soft tissue changes. Hence, it may also be useful in diagnosing TB otitis media. However, differentiating the bone and soft tissue changes in TBOM against those seen in non-tuberculous otitis media is not easy. In fact, the diagnosis of TBOM, as mentioned in most reports, is often in accordance with the clinical, and, hardly on the radiographic aspects of the disease. ${ }^{30}$ Some radiographic findings in TBOM are similarly seen in non-TB otitis media such as ossicular destruction, sclerosis of the mastoid cortex and opacification of the middle ear and mastoid. A recent study showed that a group of patients with confirmed diagnosis of TBOM had soft tissue attenuation in the entire middle ear cavity, preservation of the mastoid air cells without sclerotic change and soft tissue extension to the external auditory canal (EAC) or mucosal thickening of the bony EAC that were statistically different from non-TB chronic otitis media cases $\left(\mathrm{x}^{2}\right.$ test, $\left.\mathrm{P}<0.05\right)$. On the other hand, ossicular and scutum erosion were found significantly less in TBOM cases than chronic otitis media cases with cholesteatoma formation ( $\mathrm{x}^{2}$ test, $\left.\mathrm{P}<0.05\right)(30)$. These findings are exemplified by the CT images of twelve patients included in our local study (Figure 3).

The diagnostic tools for identifying both latent and active TB infection have changed little for decades. ${ }^{3} \mathrm{~A}$ common, simple and inexpensive diagnostic procedure for suspected TBOM case is the tuberculin skin test (TST) or Mantoux test. Although TST is highly sensitive, it has poor specificity. TST antigens are often shared by other mycobacteria, including $\mathrm{M}$. bovis and an estimated $8 \%$ of Bacille Calmette Guerin (BCG)-vaccinated individuals develop false positive results. ${ }^{3}$ An area of induration 6-10 mm evaluated $48-72$ hours of 5 tuberculin units $(0.1 \mathrm{ml})$ intradermal injection of $\mathrm{M}$. TB purified protein derivative (PPD) is typically produced among those who were previously sensitized to TB bacilli, therefore, not conclusive of present TB infection. ${ }^{3}$ A positive induration of $15 \mathrm{~mm}$, however, is considered highly suggestive of TB infection 


\begin{tabular}{|c|c|c|}
\hline \multirow[t]{2}{*}{ Typical axial and coronal CT scan images of patients in our local study } & \multicolumn{2}{|c|}{ CT scan findings in thirteen ears } \\
\hline & Description & No. \\
\hline & $\begin{array}{l}\text { Soft tissue densities } \\
\text { occupying mastoid and } \\
\text { middle ear cavities }\end{array}$ & $12 / 13$ \\
\hline & $\begin{array}{l}\text { Preserved mastoid air cell } \\
\text { architecture with minimal or } \\
\text { absent mastoid cortex sclerosis }\end{array}$ & $12 / 13$ \\
\hline & Absent ossicular bone necrosis & $10 / 13$ \\
\hline & Absent scutum blunting & $12 / 13$ \\
\hline & $\begin{array}{l}\text { Soft tissue extension over bony } \\
\text { external auditory canal }\end{array}$ & $5 / 13$ \\
\hline
\end{tabular}

Figure 3. CT scan findings in twelve patients (thirteen ears) included in the local study.

than the result of BCG vaccination. ${ }^{6}$ All patients in our series were older than 18 years old and almost all registered skin induration of $15 \mathrm{~mm}$, or greater, after TST. In developed countries and other countries with low prevalence of TB, it is likely that positive TST in adults indicate TB infection. Hence, non-responsiveness to adequate standard antibiotic therapy for bacterial otitis media in a patient with positive test should prompt the clinician to pursue confirmatory test for TBOM. In contrast, because of the likelihood of false positive tuberculin test in less developed countries considered as high burden areas for TB, the more stringent criterion of $>15 \mathrm{~mm}$ induration in a similar patient as indicative of TBOM may be applied. A new diagnostic test that assays interferon gamma, a cytokine released by responding $\mathrm{T}$ helper cells at the site of $\mathrm{TB}$ infection, has recently been introduced in the United States. Neither tuberculin nor interferon-gamma assay, however, is found capable of differentiating between latent and active infection. ${ }^{3}$

Chest radiography is considered a sensitive but nonspecific test for tuberculosis. ${ }^{6}$ Reports confirm the frequent, yet variable, association of positive chest findings to TBOM in $14-95 \%$ of cases. ${ }^{17-20}$ Chest radiograph findings are sensitive but non-specific and should not be used solely as the diagnostic criterion for the presence of TB infection. It is most useful when applied as part of a systematic approach in the evaluation of a person whose signs and symptoms suggest TBOM. ${ }^{6}$ In our local series, six of twelve cases had positive chest $\mathrm{X}$-ray described as pulmonary infiltrates and granuloma formation in five and miliary TB in one. One patient who had no pulmonary symptoms even presented with miliary pulmonary involvement on chest radiograph. It is recommended that in order to ascertain the correlation of active pulmonary involvement with TBOM, CT of the thorax should be done. ${ }^{10}$ In areas where the prevalence of TB is low, only $15-20 \%$ of extrapulmonary TB cases have lung involvement. In contrast, in high burden areas of $\mathrm{TB}$, the proportion of cases with concomitant lung findings is higher. ${ }^{6}$ This explains the wide variability of chest findings among TBOM cases, as reported in the literature. This is confirmed by our findings of $50 \%$ positive chest $x$-ray in cases with TBOM, one of whom had even undiagnosed miliary TB.

Microbiological diagnosis is necessary to establish the diagnosis of TB. The three frequent methods of microbiological diagnosis are by culture, microscopy and by nucleic acid amplification test (NAAT) or polymerase chain reaction test for $\mathrm{TB}$ (PCR $\mathrm{TB}$ ). The gold standard for bacteriologic diagnosis is by culture, either by using a solid medium such as Lowenstein-Jensen and Ogawa media or, by using liquid media systems like the BACTEC or MGIT systems. Solid media are more popular because they are less technically demanding and may be made locally. On the other hand, there is less delay in obtaining the results from liquid media. The disadvantages of culture are its cost, technical complexity and the delay in obtaining the result, 
which usually takes one month. As an alternate to culture and which is, in fact, considered the first bacteriologic test of choice is microscopic demonstration of acid-fast bacilli (AFB stain). It is technically simple, cheap and considered the most rapid method of verifying the presence of TB bacilli. However, the success of microscopy depends upon the concentration of bacilli in the specimen. Whereas a properly done culture can detect TB with about 100 organisms per ml in the specimen, microscopy needs 10,000 organisms per $\mathrm{ml}$ for positive TB detection. Because there are generally less TB organisms in extra pulmonary TB specimen, identification of acid-fast bacilli by microscopy in specimen obtained from extra pulmonary sites is less frequent. ${ }^{6}$ This explains why AFB stain was positive for bacilli in only one of seven specimens in our series. ${ }^{14}$ Nucleic acid amplification test (NAAT) or PCR TB is gaining popularity as confirmatory test for extra-pulmonary $\mathrm{TB}$ due to its ability to provide more quick results than culture methods. ${ }^{6}$ However, though it has high specificity, it also lacks sensitivity ${ }^{31}$ particularly in smear negative specimens, is not widely available in less developed areas, and is relatively expensive. Nevertheless, its main advantage over microscopy and culture is that it may detect presence of TB bacilli in specimen with fewer number of organisms due to its ability to amplify minute amounts of DNA from clinical specimens. In our study, detection rate for PCR (83\%) was higher than culture and microscopy. In another local study in patients with meningitis, PCR was considered a promising alternative to improve the yield of conventional diagnostic methods in detecting TB infection. ${ }^{32}$

Histopathological examination of specimen is being advocated as confirmatory test for specimen obtained from extrapulmonary sites. ${ }^{6}$ The typical pathologic finding for TBOM is the presence of tubercles in the undersurface of the tympanic membrane, middle ear and mastoid mucosa ${ }^{21}$ which results after establishment of TB infection through either hematogenous spread, lymphatic dissemination or by direct extension from the nasopharynx through the eustachian tube. ${ }^{33}$ In general, the transmission of $\mathrm{TB}$ is almost exclusively via the airborne route and extrapulmonary TB resulting from contiguous spread usually presents with focal findings such as in otitis media. On the other hand, hematogenous dissemination results in multisystem miliary disease. ${ }^{6}$ If the focal lesion in the middle ear is established, the tubercles can cause multiple or single eardrum perforation, infiltrate the underlying bone, erode the fallopian canal and produce facial nerve paralysis, damage the ossicular structures, infiltrate the oval or round window and cause labyrinthitis and produce other complications related to bone erosion. The basic histologic picture of $\mathrm{TB}$ is the caseous foci that is surrounded by epithelioid or histiocytic cells, multinucleated giant cells mixed with acute and chronic inflammatory cells. ${ }^{33}$ The yellowish-white caseous foci are composed of mycobacterial lipids, proteins and cellular debris which develops into the center of the tuberculoid granuloma. The surrounding histiocytic cells are actually transformed macrophages and monocytes that initially ingested the M. TB bacilli and which were subsequently transformed by cytokines released by activated T-helper lymphocytes in order to possess bactericidal and bacteriostatic capabilities. The typical gross appearance of the TB granuloma on biopsy is that of pale granulation tissue with whitish to yellowish components. A sample of this tissue may be spread over a glass slide where one may notice a clear separation of the reddish granulation tissue component and the whitish component which probably represents the area of caseation necrosis (Figure 4) (Personal experience - GTA). In an immunocompetent host, the caseous foci may exist as solid lesions, may be resolved by cell-mediated immune response, and are considered latent tuberculous infection in $95 \%$ of cases. ${ }^{6}$ Though $\mathrm{M}$. tuberculosis may survive in these solid caseous lesions their replication is inhibited by the diminished oxygen content, reduced $\mathrm{pH}$, and the accumulation of local fatty acids; thus represent latent and non-infectious lesions. In contrast, the tuberculous organism may not be contained by an immunecompromised host and the caseous lesion may exist as liquefied caseum where the TB bacilli may replicate and remain infectious. ${ }^{6}$ Since most inflammatory conditions of the temporal bone manifest histologically with acute and chronic inflammation, it is quite important for the clinician to alert the pathologist on the possibility of TB infection for the latter to take efforts in looking for the typical histologic features of TB. Given the low yield of microscopy and culture in the diagnosis of TBOM, histopathologic examination of the specimen and PCR may be considered as rational alternatives, as shown in our local study. ${ }^{14}$

The confirmatory tests for TB like TB culture, AFB microscopy, biopsy and PCR TB examination are known to be poorly sensitive with high specificity. In interpreting the results of these diagnostic tests, as in other diagnostic tests with known high specificity, a positive result should be interpreted as highly favorable of positive TB infection (SPin). On the other hand, because of their low sensitivity, highly sensitive tests like clinical examination, tuberculin skin test, chest X-ray, and temporal bone CT must be done initially since negative results in these tests may practically rule out TB infection (Snout). ${ }^{34,35}$ In other words, the diagnosis of TB otitis media, like most other diseases, follows the traditional pattern of doing good clinical examination in conjunction with relevant diagnostic tests.

\section{Review of treatment}

\section{Medical therapy:}

The mainstay of medical treatment of $\mathrm{TB}$ during the past three decades was daily isoniazid (INH). ${ }^{36}$ A Large number of well-designed clinical trials have recently pro 


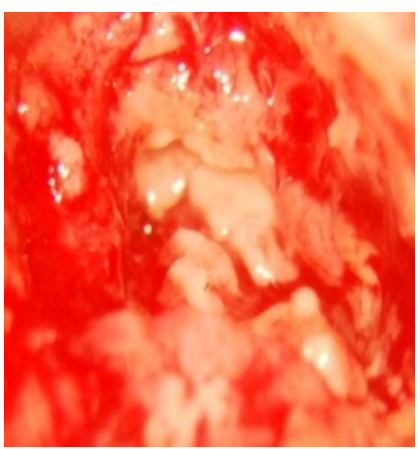

A

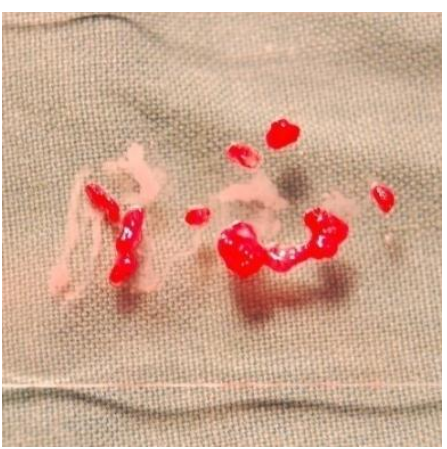

B

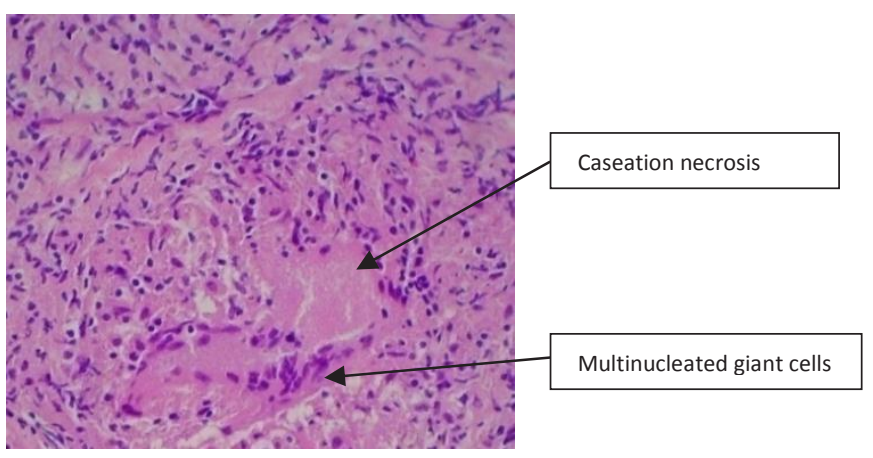

C

Figure 4. Granulation tissue with cheesy, whitish component (A) taken from the mastoid cavity. The whitish component separates from the red granulation tissue component when placed and spread over a glass slide (B). The typical histopathologic findings are shown in C.

vided the data that a rifampicin-containing regimen is the backbone of antiTB chemotherapy. ${ }^{6}$ In the Philippines, the therapeutic regimen recommended for extra-pulmonary TB consist of four drug therapy with isoniazid (INH) 400mg per day, rifampicin (RF) 600mg per day, pyrazinamide (PZA) 2 grams per day, and ethambutol (EMB) 15mg per $\mathrm{kg}$ per day immediately followed by seven month triple therapy of INH, RF and PZA. ${ }^{37}$ Newly published standards for treatment for all forms of TB consist of a shorter six month course of two month quadruple therapy with RF, INH, PZA and EMB and four month continuation phase consisting of RF and INH, preferably in fixed-dose combinations. ${ }^{6}$ In our series, a nine month treatment regimen as prescribed by the Task Force on Tuberculosis in the Philippines was followed, consisting of two month quadruple therapy followed by a three drug combination (INH, RF, PZA) for seven months was given to ten patients and for ten months to a patient who had miliary pulmonary TB. ${ }^{37}$

\section{Surgical intervention:}

The role of surgery in the treatment of TB otitis media remains controversial. Some authors thought that the role of surgery is mainly to obtain the specimen for confirmatory diagnosis and that mastoidectomy for the purpose of eradicating the disease is not indicated since complete resolution of the granuloma and otorrhea is possible by proper antituberculous chemotherapy alone. ${ }^{17}$ On the other hand, others believed that medical treatment is not enough and that surgery is necessary because bone destruction is often a rapid and early feature of $\mathrm{TB}$ otitis media and that facial palsy is possible even after the commencement of appropriate anti-TB chemotherapy. ${ }^{38}$ In our study, it was shown that regardless of the type of middle ear reconstructive procedure, there was not much difference in treatment outcomes clinically, radiologically and audiometrically. ${ }^{14}$ Generally, the included patients had variable degree of hearing improvement depending on the severity of the disease (Figure 5). Those who had more complicated procedures like tympanomastoidectomy with ossiculoplasty had similar results as those who had tympanoplasty alone. On the other hand, those who had biopsy of an accidentally detected nasopharyngeal mass on CT scan and which was confirmed histologically as TB and those who had myringotomy alone with PCR TB diagnosis of the middle ear effusion had significantly different treatment outcomes compared to patients who had mastoid and middle ear reconstructive procedures. By contrast, a patient whose diagnosis was missed during the first surgical procedure and who later underwent revision mastoidectomy and middle ear reconstructive surgery, had profound hearing loss despite good surgical outcome in terms of tympanic membrane and ossicular graft survival. These findings emphasize not only the importance of early diagnosis $^{14}$ but further concur with previous opinions ${ }^{17,19}$ stating that surgery is mainly helpful in obtaining confirmatory diagnosis and that patients who were diagnosed early may have excellent treatment outcomes, if given the proper chemotherapeutic regimen. These findings raise the issue as to the need for doing specimen confirmatory diagnosis before deciding on a definitive mastoid or middle ear reconstructive procedure, for which these opinions are still lacking. Because of the limited number of patients in our local series, we are not in a position to make an opinion regarding the role of surgery in preventing facial nerve paralysis and other complications particularly among those patients with severe disease since most patients in our study were probably diagnosed in the early stage of the disease.

\section{Conclusion/Summary}

The ubiquitous character of tuberculosis is exemplified not only in its pulmonary form but also its extrapulmonary form like TB otitis media. Our review indicates that the 

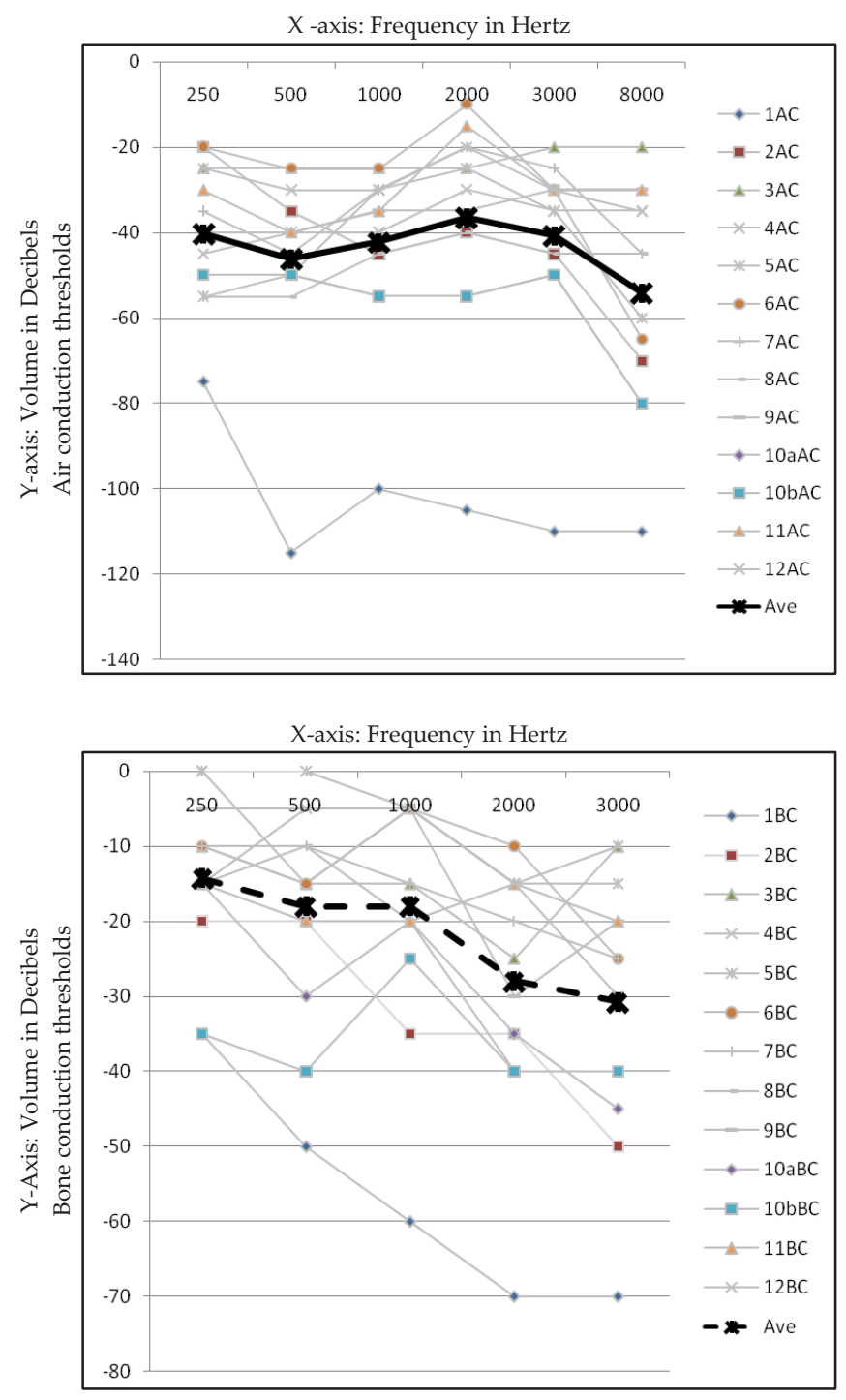

Figure 5. Individual post-treatment air and bone thresholds (light lines). The average thresholds are designated by bold lines.

clinical manifestations are not in accordance with classical descriptions in concurrence with other observations. It is likely that the pathophysiology of middle ear TB infection commonly results from direct extension of the infectious process in the nasopharynx through the eustachian tube, similar to the more common non-tuberculous otitis media. It is quite understandable, therefore, that except for patients who present with eardrum multiple perforations and masslike middle ear lesions, the otoscopic appearances of nontuberculous and TB otitis media are quite similar. This underlines the need for including nasopharyngeal examination in the routine physical examination of a suspected case. The lack of typical clinical presentation of TBOM puts emphasis on the need for suspecting the disease among those who had failed or poor treatment response to the standard purulent otitis media regimen. A strong suspicion should prompt the clinician to obtain more information on possible acquisition of the disease not only from members of the family but from peers in the workplace. Positive chest radiography, tuberculin skin test and high resolution temporal bone CT scan results should be treated as indicative signs of TB otitis media. CT scan requests should include views of the nasopharynx, and if a mass is confirmed, biopsy specimen should be submitted for histopathological study, TB culture and PCR TB examination. Samples of ear discharge among patients who present with otorrhea may be sent for TB culture and PCR TB examination. Patients who present with middle ear effusion may undergo myringotomy and PCR and TB culture examination of the collected middle fluid. Mastoid and/or middle ear granulation tissues must be examined by biopsy, TB culture and PCR TB test. Since the available confirmatory tests for TB are highly specific but insensitive, diagnosis of TBOM needs good history taking and physical examination in conjunction with relevant laboratory testssimilar to the tried and tested diagnostic processes clinicians frequently do for many clinical disorders. There are indications that the role of surgery is mainly to establish the diagnosis particularly for cases which are detected in the early stage of the disease since standard chemotherapeutic regimens were found effective in obtaining excellent clinical outcomes, even without complicated mastoid and middle ear reconstructive procedures. The clinical challenge therefore is to detect the disease early because of its profound effects on treatment outcomes.

\section{References}

1. Rubin SA. Tuberculosis - Captain of all these men of death. Radiol Clin North Am. 1995; 33(4): 619-39.

2. World Health Organization. Global tuberculosis control. Surveillance, planning, financing. WHO Report 2005.

3. Harries AD. Tuberculosis and human immunodeficiency virus infection in developing countries. Lancet. 1990; 335(8686):387-90.

4. Hernandez C, Cetner AS, Jordan JE, Puangsuvan SN, Robinson JK. Tuberculosis in the age of biologic therapy. J Am Acad Dermatol. 2008; 59(3):363-80.

5. Centers for Disease Control and Prevention. National Center for HIV/AIDS, viral hepatitis, STD, and TB prevention, Division of Tuberculosis Elimination. Surveillance Reports: Reported Tuberculosis in the United States [Online]. 2005 [cited 2010 Dec]. Available from http://www.cdc.gov/tb/surv/2005/pdf/table 28.pdf.

6. Tuberculosis Coalition for Technical Assistance. International Standards for Tuberculosis Care. Second edition. Tuberculosis Coalition for Technical Assistance. The Hague. 2009.

7. Hopewell PC. A clinical review of tuberculosis. Radiol Clin North Am. 1995; 33(4):641-53.

8. Al-Serhani AM. Mycobacterial infection of the head and neck: presentation and diagnosis. Laryngoscope. 2001; 111(11 Pt 1):2012-6.

9. Prasad KC, Sreedharan S, Chakravarthy Y, Prasad SC. Tuberculosis in the head and neck: experience in India. J Laryngol Otol. 2007; 121(10):979-85. 
10. Chirch LM, Ahmad K, Spinner W, Jimenez VF, Donelan SV, Smouha E. Tuberculous otitis media: report of 2 cases on Long Island, N.Y., and a review of all cases reported in the United States from 1990 through 2003. Ear Nose Throat J. 2005; 84(8):488-92.

11. Cantwell MF, Snider DE Jr, Cauthen GM, Onorato IM. Epidemiology of tuberculosis in the United States, 1985 through 1992. JAMA. 1994; 272(7):535-9.

12. Awan MS, Salahuddin I. Tuberculous otitis media: two case reports and literature review. Ear Nose Throat J. 2002; 81(11):792-4.

13. Brutoco RL, Spencer MJ. Tuberculous otomastoiditis: an old disease renewed. West J Med. 1980; 133(1):69-71.

14. Abes GT, Abes FL, Jamir JC. The variable clinical presentation of tuberculous otitis media and the importance of early detection. Otol Neurotol. 2011; 32(4):539-43.

15. Turner AL, Fraser JS. Tuberculosis of the middle ear cleft in children: a clinical and pathological study. J Laryngol Rhinol Otol. 1915; 30:209-47.

16. Samuel J, Fernandes CM. Tuberculous mastoiditis. Ann Otol Rhinol Laryngol. 1986; 95(3 Pt 1):264-6.

17. Singh B. Role of surgery in tuberculous mastoiditis. J Laryngol Otol. 1991; 105(11):907-15.

18. Ramages LJ, Gertler R. Aural tuberculosis: a series of 25 patients. J Laryngol Otol. 1985; 99(11):1073-80.

19. Palva T, Palva A, Karja J. Tuberculous otitis media. J Laryngol Otol. 1973; 87(3):253-61.

20. Plester D, Pusalkar A, Steinbach E. Middle ear tuberculosis. J Laryngol Otol. 1980; 94(12):1415-21.

21. Schuknecht HF. Pathology of the Ear, $2^{\text {nd }}$ printing. Cambridge, MA: Harvard University Press; 1976.

22. Vaamonde P, Castro C, Garcia-Soto N, Labella T, Lozano A. Tuberculous otitis media: a significant diagnostic challenge. Otolaryngol Head Neck Surg. 2004; 130(6):759-66.

23. Windle-Taylor PC, Bailey CM. Tuberculous otitis media: a series of 22 patients. Laryngoscope. 1980; 90(6 Pt 1):1039-44.

24. Sahn SA, Davidson PT. Mycobacterium tuberculosis infection of the middle ear. Chest. 1974; 66(1):104-6

25. Sonmez G, Turhan V, Senol MG, Ozturk E, Sildiroglu HO, Mutlu H. Relationship between tuberculous otomastoiditis and tuberculous meningitis. J Laryngol Otol. 2008; 122(9):893-7.

26. Sens PM, Almeida CI, Valle LO, Costa LH, Angeli ML. Tuberculosis of the ear, a professional disease? Braz J Otorhinolaryngol. 2008; 74(4):6217
27. Nicolau Y, Northrop C, Eavey R. Tuberculous otitis in infants: temporal bone histopathology and clinical extrapolation. Otol Neurotol. 2006; 27(5):667-71.

28. Abes GT. Signs and symptoms of TB otitis media in 12 patients. 2010. Unpublished.

29. Conly JM, Winger MJ, Low DE, Harding GK. Tuberculous otitis media: an old disease revisited. Can Med Assoc J. 1985; 132(5):512-3.

30. Rho MH, Kim DW, Kim SS, Sung YS, Kwon JS, Lee SW. Tuberculous otomastoiditis on high resolution temporal bone CT: comparison with non-tuberculous otomastoiditis with and without cholesteatoma. AJNR Am J Neuroradiol. 2007; 28(3):493-6.

31. Pai M, Flores LL, Pai N, Hubbard A, Riley LW, Colford JM Jr. Diagnostic accuracy of nucleic acid amplification tests for tuberculous meningitis: a systematic review and meta-analysis. Lancet Infect Dis. 2003; 3(10):63343.

32. Pasco PM, Pascual JL, Handumon JC. Use of polymerase chain reaction in the diagnosis of tuberculous meningitis. Acta Med Philipp. 2007; 41:68-71.

33. Hyams VJ. Granulomatous disorders of the temporal bone. Otolaryngol Clin North Am. 1982; 15(3):515-8.

34. Sackett DL, Straus SE, Richardson WS, Rosenberg WM, Haynes RB. Evidence-based Medicine: How to practice and teach EBM, $2^{\text {nd }}$ ed. London: Churchill Livingstone; 2000.

35. Sackett DL. The rational clinical examination. A primer on the precision and accuracy of the clinical examination. JAMA. 1992; 267(19):2638-44.

36. Screening for tuberculosis and tuberculosis infection in high-risk populations Recommendations of the Advisory Council for the Elimination of Tuberculosis. MMWR Recomm Rep. 1995; 44(RR-11):1934.

37. Task Force on Tuberculosis, Philippine Practice Guidelines Group in Infectious Diseases. Pulmonary Tuberculosis: Clinical Practice Guidelines. PPGG-ID Philippine Society for Microbiology and Infectious Diseases and Philippine College of Chest Physicians, 2000.

38. Weiner GM, O'Connell JE, Pahor AL. The role of surgery in tuberculous mastoiditis: appropriate chemotherapy is not always enough. J Laryngol Otol. 1997; 111(8):752-3. 